\title{
Improved comprehensive analytical method for assessment of satranidazole in drug and product
}

\author{
Rana Mazumder ${ }^{1,2^{*}}$, Beduin Mahanti ${ }^{1}$, Subhabrota Majumdar ${ }^{2}$, Rabindranath Pal ${ }^{2}$ and Ashok Dhar Chowdhury ${ }^{2}$
}

\begin{abstract}
Background: The aim of the present investigation was to improve comprehensive analytical method for the assessment of satranidazole in drug and product with simple, economic, sensitive, and reproducible spectrophotometric method. The drug was analyzed in three different methods by using various solvents where satranidazole (STZ) showed different absorbance maxima (s) and sharp peaks in the first order derivative spectra. Beer's law range, correlation coefficient, apparent molar absorptivity, etc., were determined for each solvent using all the three methods.
\end{abstract}

Results: All the results of analysis were found to be satisfactory which was validated statistically for various parameters and by performing recovery studies in accordance with international conference on harmonization (ICH) guidelines. The developed methods were also compared statistically using one way analysis of variance (ANOVA).

Conclusion: Three different spectrophotometric methods were developed for satranidazole (STZ) using various inorganic and organic solvents. The analytical methods are found to be simple, sensitive, rapid, specific, and economic, and it can be conveniently employed for the routine analysis, quality control. The sample recoveries from the formulation were in good agreement with its respective label claim.

Keywords: Spectrophotometric, Satranidazole (STZ), ICH guidelines, Absorptivity, Analysis of variance (ANOVA), Validation

\section{Background}

Satranidazole (STZ) is a new drug in the class of nitroimidazole derivative compounds. It is chemically known as 1-methylsulfonyl-3-(1-methyl-5-nitro-2-imidazolyl)-2imidazolidinone and therapeutically is a potential antibacterial and antiprotozoal which used management of amoebiasis [1]. It is also effective against E. hystolytica, $T$. vaginalis, and Giardia. At the 2-position, the substituted (methyl sulphonated) imidazolidinone has been found to produce more activity against microaerophilic and

\footnotetext{
* Correspondence: ranapharma.mazumder@gmail.com

'School of Pharmacy, Techno India University, EM 4, Sector-V, Kolkata, West Bengal 700091, India

${ }^{2}$ Department of Pharmaceutics, Calcutta Institute of Pharmaceutical Technology \& Allied Health Sciences, Banitabla, Uluberia, Howrah, West Bengal 711316, India
}

\section{Springer Open}

(c) The Author(s). 2020 Open Access This article is licensed under a Creative Commons Attribution 4.0 International License, which permits use, sharing, adaptation, distribution and reproduction in any medium or format, as long as you give appropriate credit to the original author(s) and the source, provide a link to the Creative Commons licence, and indicate if changes were made. The images or other third party material in this article are included in the article's Creative Commons licence, unless indicated otherwise in a credit line to the material. If material is not included in the article's Creative Commons licence and your intended use is not permitted by statutory regulation or exceeds the permitted use, you will need to obtain permission directly from the copyright holder. To view a copy of this licence, visit http://creativecommons.org/licenses/by/4.0/. fourfold lower than metronidazole [2]. Study of different literature shows that satranidazole exhibits remarkable higher plasma concentration in comparison with metronidazole. Satranidazole shows $1.01 \mathrm{~h}$ of plasma elimination half life in comparison with metronidazole with $3.62 \mathrm{~h}$. In addition to that the report of tolerance of satranidazole was significantly better than metronidazole was significantly better than metronidazole and adverse effect incidents are potentially low in satranidazole. Thus, although both the drugs having similar efficacy, use of satranidazole is more advantageous with lower incidence of side effect and better tolerance [3].

Satranidazole (STZ) is a light yellow crystalline powder, odorless, and bitter in taste. The powder has high melting point $\left(186{ }^{\circ} \mathrm{C}\right)$, loss on drying at $105^{\circ} \mathrm{C}$ after $3 \mathrm{~h}$ 
was found not more than $0.5 \%$. Estimation of satranidazole (STZ) was carried out in different type of aqueous, organic and inorganic solvents which includes double distilled water (DDW), different buffer solutions and acidic/basic solutions. Organic solvents may be classified in the following three classes-polar, semipolar and nonpolar solvents. Polar solvents are of two types: polar protic and polar aprotic, and they are named as such depending on their dissolution mechanism. The former one uses $\mathrm{H}$ bonding where the latter one uses a high "di pole-di pole" interface for solubilization. Methanol, ethanol, isopropyl alcohol, and glacial acetic acid are few examples of polar-protic solvents, and dimethyl formamide (DMF), acetonitrile, methelen chloride, etc., are polar aprotic solvents used over here. Benzene, cyclohexane, diethylether, etc., could be used as non-polar solvents, as they use vander Waals force of attraction to solubilize. Selection of solvent was done purely depending on their ease of availability cost wide acceptance as UV solvents and solubility of satranidazole. Solvents found giving peaks in the near UV region were corrected via resetting the scanning range with suitable lower limit.

Several study showed that validation and estimation of STZ may be done using high-performance liquid chromatography (HPLC) [4], high-performance thin layer chromatography (HPTLC) [5, 6] colorimetric method after chemical derivatization $[7,8]$, and UVspectrophotometric methods both in combination with ofloxacin or gatifloxacin or alone $[9,10]$. Absence of sufficient experimental data of UVspectrophotometric methods for quantitative estimations of satranidazole (STZ) in pharmaceutical formulation using different classes of solvents influence to perform the present study. The purpose of validating an analytical procedure as per $\mathrm{ICH}$ guidelines are identification or recognizable proof tests, quantitative evaluations for impurity, limit tests for control of contaminations, and quantitative trial in substance or selected component. Distinctive validation parameters considered were accuracy, precision, specificity, limit test, quantization, and linearity to be assessed. A revalidation is also important according the nature of change, i.e., changes in the synthesis, composition, and analytical procedure.

\section{Methods}

UV-visible spectrophotometer-1700 (Simadzu, Japan) with spectral band width of $2 \mathrm{~nm}$, wavelength with accuracy of $\pm 0.5 \mathrm{~nm}$ and a pair of absorbance was measured using $1 \mathrm{~cm}$ matched quartz cells.

Satranidazole gift sample was kindly provided by Alkem Laboratories Limited, Baddi, India. Satranidazole tablets (SATROGYL, $300 \mathrm{mg}$ satranidazole, manufactured by Alkem Laboratories Limited, East Sikkim,
India) was purchased from local pharmacy. Methanol, ethanol, dichloromethane, N, N-dimethylformamide, acetonitrile, and benzene were purchased from Sigma Lab, Mumbai, India.

\section{Preparation of standard stock solution}

Solutions containing $500 \mu \mathrm{g} \mathrm{ml}^{-1}$ of pure drug were prepared by dissolving $25 \mathrm{mg}$ of STZ in sufficient solvent, and volume was adjusted up to $50 \mathrm{ml}$ in a volumetric flask. The solutions are sonicated till half an hour (especially when double distilled water and benzene were taken as solvent). From these, aliquots solutions were pipette out and diluted with same solvent to obtain standard stock solutions of $100 \mu \mathrm{g} \mathrm{m}{ }^{-1}$ satranidazole (STZ) concentration.

\section{Procedure for calibration curve Method a: absorption maxima method ( $\left.\lambda_{\max }\right)$}

Ten micrograms per milliliter solution of satranidazole (STZ) was prepared by diluting the standard stock solution. Subsequent scanning was done 200$400 \mathrm{~nm}$. The shift of $\lambda_{\max }$ value of satranidazole (STZ) in different medium described as "solvent effect" [11]. Baseline correction cannot always compensate the unacceptable effects in spectra recording [12]. Solvents showing interference with maximum absorption region were excluded, i.e., acetone, glacial acetic acid. Concentration of the solution can be determined by calibration curve.

\section{Method B: first order derivative method}

Ten micrograms per milliliter solution of satranidazole (STZ) was prepared by using the same method described under method A and scanned in the range of 400-200 $\mathrm{nm}$ using different solvents. The first derivative spectra $\left(D^{1}\right)$ are the rate of change of absorbance against wavelength $\left(\frac{\mathrm{dA}}{\mathrm{d} \lambda} \mathrm{vs} \lambda\right)$. This process is one of the modern spectrophotometric techniques that are useful for gathering qualitative and quantitative information from the above spectra and overlapped bands. This method helps to quantify one analyte without separation in primary phase [13]. The sharp peak chosen for each solvent was used for quantification respectively. From the spectra of drug maxima values were listed in Table 1 . Concentration of sample solution was determined by using the calibration curve.

\section{Method C: AUC method}

Ten micrograms per milliliter solution of satranidazole (STZ) was prepared by using the same method described under method $\mathrm{A}$ and scanned in the range of $200-400 \mathrm{~nm}$ using different solvents. Area under 
Table 1 Report of UV-spectroscopic characteristics for satranidazole (STZ) in various solvents

\begin{tabular}{|c|c|c|c|c|c|c|c|c|c|}
\hline \multirow[t]{2}{*}{ SLNo } & \multirow[t]{2}{*}{ Solvent used } & \multirow{2}{*}{$\begin{array}{l}\text { Cut- } \\
\text { off } \\
(\mathrm{nm})\end{array}$} & \multirow{2}{*}{$\begin{array}{c}\lambda_{\text {Max }}^{D 0} \\
(\mathrm{~nm})\end{array}$} & \multirow{2}{*}{$\begin{array}{l}\Delta \lambda^{b} \\
(\mathrm{~nm})\end{array}$} & \multirow{2}{*}{$\begin{array}{l}\lambda_{\mathrm{Max}}^{D 1} \\
(\mathrm{~nm})\end{array}$} & \multirow{2}{*}{$\begin{array}{l}\text { AUC } \\
(\lambda 1-\lambda 2)\end{array}$} & \multicolumn{3}{|l|}{ Linearity } \\
\hline & & & & & & & A: Method & B: Method & C: Method \\
\hline 1. & Double distilled water & 205 & 320 & 1 & 293 & $325-315$ & $5-30$ & $5-35$ & $5-30$ \\
\hline 2. & Methanol & 210 & 316 & 1 & 290 & $320-313$ & $5-25$ & $5-35$ & $5-25$ \\
\hline 3. & Ethanol & 210 & 316 & 1 & 294 & $320-312$ & $5-40$ & $5-60$ & $5-40$ \\
\hline 4. & DMF & 268 & 329.8 & 1 & 306 & $334-324$ & $5-15^{\mathrm{a}}$ & $5-20^{\mathrm{a}}$ & $5-15^{\mathrm{a}}$ \\
\hline 5. & Benzene & 280 & 320 & 1 & 300.5 & $322-317$ & $5-30$ & $5-40$ & $5-30$ \\
\hline 6. & Acetonitrile & 210 & 325 & 1 & 297.5 & $328-320$ & $5-10^{a}$ & $5-15^{\mathrm{a}}$ & $5-10^{a}$ \\
\hline 7. & Dichloro methane & 235 & 322 & 1 & 296 & $327-318$ & $5-25$ & $5-40$ & $5-25$ \\
\hline
\end{tabular}

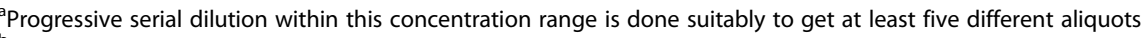

${ }^{b}$ For derivative spectra

the curve (AUC) can be determined by following equation:

$$
\mathrm{AUC}=\int_{\lambda_{\mathrm{x}}}^{\lambda_{\mathrm{y}}} \operatorname{Ad} \lambda=\alpha+\beta
$$

$\lambda_{\mathrm{y}}, \lambda_{\mathrm{x}}=$ starting and ending wavelength.

$\alpha=$ area of segment surrounded by curve data and a straight line between the start and end point.

$\beta=$ area of segment surrounded by a straight line between the start and end point on curve data and horizontal axis.

Area under the curve (AUC) method [14] includes the calculation of incorporated estimation of absorbance as for wavelength. Here, the horizontal axis corresponds to base line. The wavelength ranges were selected on the basis of repeated observations expressing better linearity given by area under the curve (AUC) of variety of wavelength ranges vs. concentration. Calibration curves were plotted by the respective area under the curve (AUC) values vs. concentrations.

\section{Method for product analysis}

Here, 20 products as tablets were weighed, and average weight was taken for estimation of drug in the formulations. After crushing the tablets, fine powder was collected equivalent to $50 \mathrm{mg}$ satranidazole (STZ) which transferred to $100 \mathrm{ml}$ of volumetric flask. Ultrasonicated solvent was used to make up the volume. Filtration of solutions was done by using Whatman filter paper and from it, $5 \mathrm{ml}$ of filtrate was transferred in to a 5-ml volumetric flask and diluted up to the mark. Further dilutions from this working standard are done to obtain $15 \mu \mathrm{g} \mathrm{ml} \mathrm{m}^{-1}$ of satranidazole (STZ) solution. Satranidazole concentration is determined by method A, which measured the sample absorbance in the respective absorbance maxima $\left(\lambda_{\max }\right)$ of satranidazole (STZ), found in that solvent (Table 1). The concentration of satranidazole in the sample solution was determined by using calibration curve. Similarly by method B, the corresponding wave length $\left(\lambda_{1}\right)$ in the first order derivative mode of the used solvent selected from Table 1, and absorbance was measured to determine the concentration of satranidazole (STZ) from the respective calibration curve. The suitable wavelength range for a particular solvent was chosen from Table 1 which was used to determine the concentration in method $\mathrm{C}$ from the respective calibration curve. The above methods are carried out separately to determine satranidazole (STZ) concentration in all the solvents used which is described by the Table 5 .

\section{Validation method}

\section{Specificity and selectivity}

The specificity of satranidazole arrangements $(10 \mu \mathrm{g}$ $\mathrm{ml}^{-1}$ ) was done by every medium with or without basic excipients independently. All arrangements were checked from 200 to $400 \mathrm{~nm}$ at a medium speed and checked whether any adjustment in the absorbance at individual frequency. In a different report, satranidazole (STZ) arrangements $\left(10 \mu \mathrm{g} \mathrm{ml}^{-1}\right)$ were set up in every medium independently from unadulterated medication stock and matched with the $t$ test at 95\% centrality level with consequence investigation $(n=10)$ performed to determine the absorbance (Table 2).

\section{Linearity and range}

Linearity is the capacity to get test results corresponding to the mount of analyte present [15]. To set up linearity of the proposed strategies, 10 separate arrangement of proper dilution in the range of $05-50 \mu \mathrm{g} \mathrm{ml}^{-1}$ was investigated in every dissolvable independently. Optimum dilution ranges and intervals (at least 5) are set depending upon previous trial and by visual investigation of a plot of signs as a component of analyte focus, a region of direct relationship was chosen and are more distant assessed by proper measurable strategy by estimation of 
Table 2 Validation parameters and statistical data with regression analysis of satranidazole (STZ) $(n=10)$

\begin{tabular}{|c|c|c|c|c|c|c|c|c|c|}
\hline \multirow[t]{2}{*}{ Solvent } & \multirow[t]{2}{*}{ Method } & \multicolumn{5}{|c|}{ Regression analysis } & \multicolumn{3}{|c|}{ Validation parameters } \\
\hline & & $\begin{array}{l}\text { Slope } \\
\left(S . E^{a}\right)\end{array}$ & $95 \% \mathrm{CL}^{\mathrm{b}}$ of slope & Intercept (S.E') & $95 \% \mathrm{CL}$ of icpt ${ }^{d}$ & $R^{2}$ & $95 \% \mathrm{CL}$ of te & $\begin{array}{l}\text { LOD }^{f} \\
\left(\mu \mathrm{ml}^{-1}\right)\end{array}$ & $\begin{array}{l}\mathrm{LOQ}^{\mathrm{g}} \\
\left(\mu \mathrm{g} \mathrm{ml}^{-1}\right)\end{array}$ \\
\hline \multirow[t]{3}{*}{ Methanol } & A & $\begin{array}{l}0.048 \\
(0)\end{array}$ & - & $\begin{array}{l}0.027 \\
(0.0010)\end{array}$ & $3.94 \mathrm{E}-05$ & 0.998 & 0.3804 & 0.0859 & 0.2604 \\
\hline & B & $\begin{array}{l}0.027 \\
(0.0005)\end{array}$ & $1.56 \mathrm{E}-05$ & $\begin{array}{l}0.044 \\
(0.0049)\end{array}$ & 0.000126 & 0.999 & 0.1449 & 0.4925 & 1.4814 \\
\hline & C & & 7.83E-05 & $\begin{array}{l}0.308 \\
(0.1493)\end{array}$ & 0.004484 & 0.999 & 0.0630 & 1.3961 & 4.2307 \\
\hline \multirow[t]{3}{*}{ Ethanol } & A & & 0.077072 & $\begin{array}{l}0.118 \\
(0.0078)\end{array}$ & 0.000387 & 0.999 & 0.5971 & 1.9328 & 5.9725 \\
\hline & B & $\begin{array}{l}0.015 \\
(0)\end{array}$ & $\begin{array}{l}0.337 \\
(0.0030)\end{array}$ & $\begin{array}{l}0.107 \\
(0.0007)\end{array}$ & $6.00 \mathrm{E}-05$ & 0.999 & 0.8314 & 0.4210 & 1.2758 \\
\hline & C & $\begin{array}{l}0.171 \\
(0.0064)\end{array}$ & $\begin{array}{l}0.021 \\
(0.0031)\end{array}$ & $\begin{array}{l}1.259 \\
(0.1947)\end{array}$ & 0.00616 & 0.999 & 0.5142 & 3.7901 & 11.4084 \\
\hline \multirow[t]{3}{*}{ DMF } & A & $\begin{array}{l}0.056 \\
(0.0104)\end{array}$ & 0.00032 & $\begin{array}{l}0.095 \\
(0.0227)\end{array}$ & 0.00070 & 0.999 & 0.4931 & 1.0690 & 3.2178 \\
\hline & B & $\begin{array}{l}0.044 \\
(0)\end{array}$ & - & $\begin{array}{l}0.092 \\
(0.0024)\end{array}$ & $5.91 \mathrm{E}-05$ & 0.999 & 0.0873 & 0.18 & 0.5418 \\
\hline & C & $\begin{array}{l}0.647 \\
(0.0010)\end{array}$ & 0.03354 & $\begin{array}{l}0.855 \\
(0.0700)\end{array}$ & 0.002667 & 0.999 & 0.1877 & 0.3570 & 1.0746 \\
\hline \multirow[t]{3}{*}{ Benzene } & A & $\begin{array}{l}0.037 \\
(4.6 \mathrm{E}-05)\end{array}$ & 0.00101 & $\begin{array}{l}0.040 \\
(0.0013)\end{array}$ & 0.029218 & 0.999 & 0.1116 & 0.1203 & 0.3623 \\
\hline & B & $\begin{array}{l}0.026 \\
(2.5 E-05)\end{array}$ & 0.00096 & $\begin{array}{l}0.044 \\
(0.0006)\end{array}$ & 0.025024 & 0.999 & 0.3036 & 0.0805 & 0.2495 \\
\hline & $C$ & $\begin{array}{l}0.192 \\
(3.6 \mathrm{E}-05)\end{array}$ & 0.00122 & $\begin{array}{l}0.347 \\
(0.0019)\end{array}$ & 0.06532 & 0.999 & 0.6097 & 0.0332 & 0.1002 \\
\hline \multirow[t]{3}{*}{ Acetonitrile } & A & $\begin{array}{l}0.105 \\
(0.0047)\end{array}$ & 0.000128 & $\begin{array}{l}0.013 \\
(0.0225)\end{array}$ & 0.00058 & 0.999 & 0.1675 & 0.7071 & 2.1285 \\
\hline & B & $\begin{array}{l}0.064 \\
(0.0018)\end{array}$ & $6.46 \mathrm{E}-05$ & $\begin{array}{l}0.007 \\
(0.0117)\end{array}$ & 0.00036 & 0.999 & 0.7152 & 0.6037 & 1.8174 \\
\hline & $C$ & $\begin{array}{l}0.871 \\
(0.0441)\end{array}$ & 0.001319 & $\begin{array}{l}0.087 \\
(0.2136)\end{array}$ & 0.006256 & 0.999 & 0.1083 & 0.8092 & 2.4359 \\
\hline \multirow[t]{3}{*}{ Meth chloride } & A & $\begin{array}{l}0.043 \\
(0)\end{array}$ & - & $\begin{array}{l}-0.006 \\
(0.0060)\end{array}$ & 0.00018 & 0.999 & 0.0171 & 0.4662 & 1.4033 \\
\hline & B & $\begin{array}{l}0.026 \\
(0.0004)\end{array}$ & 1.70E-05 & $\begin{array}{l}-0.039 \\
(0.0061)\end{array}$ & 0.000231 & 0.999 & 0.1455 & 0.7742 & 2.3304 \\
\hline & $C$ & $\begin{array}{l}0.410 \\
(0.0303)\end{array}$ & 0.000537 & $\begin{array}{l}-0.513 \\
(0.1474)\end{array}$ & 0.005395 & 0.999 & 0.7257 & 1.1863 & 3.5710 \\
\hline \multirow[t]{3}{*}{ DDW } & A & $\begin{array}{l}0.033 \\
(0.0011)\end{array}$ & 4.92E-05 & $\begin{array}{l}(7 \mathrm{E}-06) \\
(2.2 \mathrm{E}-06)\end{array}$ & 0.00076 & 0.999 & 0.0764 & 0.0002 & 0.0006 \\
\hline & B & $\begin{array}{l}0.021 \\
(0)\end{array}$ & - & $\begin{array}{l}0.004 \\
(5.5 \mathrm{E}-05)\end{array}$ & 0.00012 & 0.999 & 0.0245 & 0.0086 & 0.0267 \\
\hline & $C$ & $\begin{array}{l}0.281 \\
(0.0091)\end{array}$ & 0.00091 & $\begin{array}{l}-0.031 \\
(0.00124)\end{array}$ & $1.92 \mathrm{E}-05$ & 0.999 & 0.0875 & 0.0145 & 0.0451 \\
\hline
\end{tabular}

\footnotetext{
${ }^{\mathrm{a}}$ Standard error of mean

${ }^{\mathrm{b}}$ Regression coefficient slopee

'Standard error

${ }^{\mathrm{d}}$ Regression coefficient

'Theoretical values (95\% confidence limit), $t=2.225$

fDetection limit

${ }^{9}$ Quantification limit
} 
a decline line by the technique for least squares. Parameters, like slope and intercept, the sum of squares, and the coefficient of relationship were accounted for (Table 2).

$\mathrm{ICH}$ guidelines [16] depict the scope of an investigative methodology as the interim among upper and lower grouping of analyte in the example for which appropriate degree of accuracy, precision and linearity are found. Reaches for various solvents and various strategies were accounted for (Table 1).

\section{Accuracy}

The accuracy of an analytical technique communicates the closeness of understanding between the qualities found. Accuracy indicates systemic error which can be gathered here from accuracy, linearity, particularity after effects of examination of results with all around described technique (data from calibration curve), and recovery of drug substance added to the placebo (drug product). For quantitative methodologies, it is suggested at least 9 judgments covering the predefined extend for the strategy (e.g., 3 concentrations/3 replicates each) [16].

Three quartile values were determined from the calibration curve of STZ within linearity range in different solvents and various strategies independently in particular lower concentration (LC, $Q_{1}$ ), middle concentration $\left(\mathrm{IC}, \mathrm{Q}_{2}\right)$, and higher concentration $\left(\mathrm{HC}, \mathrm{Q}_{3}\right)$ were set up from free stock solution and analyzed $(n=10)$. Accuracy is resolved from rate relative error (\%RE) and mean \% recovery (Table 3). Accuracy is farther accessed by standard addition method. This involves addition of three different concentration of bulk drug (known amount) in a characteristic manner for analyzed formulation sample and equivalent total concentration is measured in each solvent and each method separately. The percentage recoveries of bulk drug are determined by using following equation and recorded in Table 4.

$$
\% \text { Recovery }=\frac{\left(C_{t}-C_{s}\right)}{C_{a}} * 100
$$

$C_{\mathrm{t}}=$ total concentration of drug by measured after standard addition.

$C_{\mathrm{s}}=$ concentration of drug in the formulation.

$C_{\mathrm{a}}=$ concentration of drug added to formulation.

\section{Precision}

ICH rules state 18 shows that the exactness of analytical technique communicates the closeness of understanding (level of dissipate) between a progression of estimations got from various examining of the equivalent homogeneous example under the recommended conditions. Precision might be considered at three levels: repeatability, transitional accuracy, and reproducibility. Repeatability reflects intra test variety, where later two reflects inside research centers and between labs varieties individually. In any event, nine conclusions over the predetermined range are endorsed to perform. Repeatability are resolved utilizing various degrees of medication focuses (same concentration levels taken in accuracy study), arranged from free stock arrangement, and analyzed $(n=$ 10). The percent relative standard deviation (\%RSD) of the anticipated fixations from the relapse condition are taken as accuracy (Table 5).

\section{Limit of detection (LOD) and limit of quantitation (LOQ)}

The location and quantification of an individual systematic method was the most reduced grouping of analyte. LOD need not to be fundamentally precisely evaluated as an accurate value yet LOQ should have reasonable precision and accuracy. Those can be resolved by different path, i.e., from visual definition, from sign to commotion proportion, from standard deviation of clear, and from adjustment line utilizing the accompanying equation [16]. Results were recorded in Table 2.

$$
\mathrm{DL} ; \mathrm{QL}=\frac{F X S D}{b}
$$

$F$ is the factor of 3.3 for DL or 10 for QL. $\mathrm{SD}=$ linear regression residual standard.

$b=$ regression line slop.

\section{Robustness}

It is a determination of capacity of an analytical method to unaffected by small but deliberated variation in procedure parameters which reflects the reliability during normal usage. Few spectroscopic conditions (changing $\lambda$, using different buffer solutions) intentionally altered to monitor whether any significantly different results are obtained or not.

\section{Statistical evaluation}

The created strategies factually contrasted utilizing onepath ANOVA with show whether any critical distinction between three techniques exists or not, so these techniques can be helpful in routine investigation of STZ both in mass medication and tablet plan (Table 6).

\section{Results}

\section{Calibration curve}

Solubility profile of satranidazole (STZ) was found to be very sparingly soluble in water, but in an extremely nonpolar solvent, like cyclohexane, diethyl ether, benzene etc. It was found to show even worst solubility, but in high as well as medium polar solvents, like alcohols, acetone, DMF, DMSO, dioxane, glacial acetic acid, 
Table 3 Accuracy and precision analysis data

\begin{tabular}{|c|c|c|c|c|c|c|c|c|}
\hline \multirow[t]{2}{*}{ Solvent } & \multirow[t]{2}{*}{ Method } & \multirow[t]{2}{*}{ Level } & \multirow{2}{*}{$\begin{array}{l}\text { Conc } \\
-\mu g / \\
m l\end{array}$} & \multicolumn{3}{|c|}{ Estimated concentrations $\left(\mu \mathrm{g} \mathrm{ml}^{-1}\right)$ by linear regression equation } & \multirow{2}{*}{$\begin{array}{l}\text { Mean \% } \\
\text { recovery } \\
( \pm \text { S.D. })\end{array}$} & \multirow{2}{*}{$\begin{array}{l}\text { Accuracy } \\
d \\
(\% \text { RE) }\end{array}$} \\
\hline & & & & Range & A.M. \pm S.D. & $\%$ RSD & & \\
\hline \multirow[t]{9}{*}{ Benzene } & \multirow[t]{3}{*}{ A } & $\mathrm{LC}^{\mathrm{a}}$ & 8.75 & $8.91-8.59$ & $8.74 \pm 0.0862$ & 0.987 & $99.912 \pm 0.986$ & -0.088 \\
\hline & & $I^{b}$ & 17.5 & $17.73-17.24$ & $17.51 \pm 0.1186$ & 0.678 & $100.098 \pm 0.677$ & 0.098 \\
\hline & & $H C^{c}$ & 26.25 & $26.71-25.99$ & $26.49 \pm 0.2013$ & 0.767 & $100.95 \pm 0.767$ & 0.95 \\
\hline & \multirow[t]{3}{*}{ B } & $\mathrm{LC}^{\mathrm{a}}$ & 11.25 & $11.36-11.17$ & $11.27 \pm 0.0635$ & 0.564 & $100.198 \pm 0.565$ & 0.198 \\
\hline & & $\mathrm{IC}^{\mathrm{b}}$ & 22.5 & $22.89-22.32$ & $22.71 \pm 0.1283$ & 0.565 & $100.934 \pm 0.570$ & 0.934 \\
\hline & & $H C^{c}$ & 33.75 & $34.42-33.07$ & $33.75 \pm 0.2690$ & 0.797 & $100.005 \pm 0.797$ & 0.005 \\
\hline & \multirow[t]{3}{*}{$C$} & $\mathrm{LC}^{\mathrm{a}}$ & 8.75 & $8.92-8.57$ & $8.75 \pm 0.0554$ & 0.634 & $100.023 \pm 0.634$ & 0.023 \\
\hline & & $I C^{b}$ & 17.5 & $17.81-17.19$ & $17.55 \pm 0.1179$ & 0.672 & $100.309 \pm 0.674$ & 0.309 \\
\hline & & $H C^{c}$ & 26.25 & $26.50-25.99$ & $26.51 \pm 0.086$ & 0.327 & $100.991 \pm 0.330$ & 0.991 \\
\hline \multirow[t]{9}{*}{ Methenchloride } & \multirow[t]{3}{*}{ A } & $\mathrm{LC}^{\mathrm{a}}$ & 7.5 & $7.61-7.42$ & $7.49 \pm 0.0575$ & 0.767 & $99.991 \pm 0.766$ & -0.009 \\
\hline & & $I^{b}$ & 15 & $15.28-14.79$ & $14.99 \pm 0.1316$ & 0.878 & $99.978 \pm 0.877$ & -0.022 \\
\hline & & $H C^{c}$ & 22.5 & $22.72-22.29$ & $22.49 \pm 0.0886$ & 0.394 & $99.959 \pm 0.393$ & -0.041 \\
\hline & \multirow[t]{3}{*}{ B } & $L C^{a}$ & 11.25 & $11.43-11.24$ & $11.34 \pm 0.0665$ & 0.587 & $100.847 \pm 0.591$ & 0.847 \\
\hline & & $I^{b}$ & 22.5 & 22.69-22.09 & $22.36 \pm 0.1149$ & 0.514 & $99.416 \pm 0.510$ & -0.584 \\
\hline & & $H C^{c}$ & 33.75 & $34.35-33.39$ & $33.95 \pm 0.2027$ & 0.597 & $100.619 \pm 0.600$ & 0.619 \\
\hline & \multirow[t]{3}{*}{$C$} & $\mathrm{LC}^{\mathrm{a}}$ & 7.5 & $7.58-7.49$ & $7.55 \pm 0.0224$ & 0.298 & $100.708 \pm 0.300$ & 0.708 \\
\hline & & $I^{b}$ & 15 & $15.05-14.68$ & $14.85 \pm 0.0692$ & 0.466 & $99.053 \pm 0.461$ & -0.947 \\
\hline & & $H C^{\mathrm{C}}$ & 22.5 & $22.93-22.13$ & $22.72 \pm 0.1969$ & 0.867 & $100.986 \pm 0.875$ & 0.986 \\
\hline \multirow[t]{9}{*}{ Acetonitrile } & \multirow[t]{3}{*}{ A } & $\mathrm{LC}^{\mathrm{a}}$ & 5.75 & $5.79-5.75$ & $5.77 \pm 0.0205$ & 0.356 & $100.516 \pm 0.357$ & 0.516 \\
\hline & & $I^{b}$ & 7.5 & $7.61-7.39$ & $7.42 \pm 0.0501$ & 0.675 & $99.03 \pm 0.668$ & -0.969 \\
\hline & & $H C^{c}$ & 9.25 & $9.34-9.14$ & $9.23 \pm 0.0622$ & 0.675 & $99.83 \pm 0.673$ & -0.170 \\
\hline & \multirow[t]{3}{*}{ B } & $\mathrm{LC}^{\mathrm{a}}$ & 5 & $5.09-4.98$ & $5.04 \pm 0.0213$ & 0.423 & $100.890 \pm 0.426$ & 0.890 \\
\hline & & $I^{b}$ & 10 & $10.17-9.85$ & $10.01 \pm 0.066$ & 0.667 & $100.180 \pm 0.668$ & 0.180 \\
\hline & & $H C^{c}$ & 15 & $15.24-14.77$ & $15.06 \pm 0.1268$ & 0.842 & $100.458 \pm 0.845$ & 0.458 \\
\hline & \multirow[t]{3}{*}{ C } & $\mathrm{LC}^{\mathrm{a}}$ & 5.75 & $5.83-5.66$ & $5.76 \pm 0.0485$ & 0.841 & $100.345 \pm 0.843$ & 0.345 \\
\hline & & $I^{b}$ & 7.5 & $7.50-7.05$ & $7.44 \pm 0.0256$ & 0.587 & $99.31 \pm 0.582$ & -0.690 \\
\hline & & $H C^{c}$ & 9.25 & $9.42-9.25$ & $9.32 \pm 0.0427$ & 0.459 & $100.801 \pm 0.462$ & 0.801 \\
\hline \multirow[t]{9}{*}{ DMF } & \multirow[t]{3}{*}{ A } & $\mathrm{LC}^{\mathrm{a}}$ & 5 & $5.04-4.99$ & $5.02 \pm 0.009$ & 0.187 & $100.409 \pm 0.187$ & 0.409 \\
\hline & & $\mathrm{IC}^{\mathrm{b}}$ & 10 & $10.12-9.92$ & $10.07 \pm 0.0247$ & 0.246 & $100.763 \pm 0.247$ & 0.763 \\
\hline & & $H C^{\mathrm{C}}$ & 15 & $15.19-14.79$ & $14.96 \pm 0.0772$ & 0.516 & $99.74 \pm 0.514$ & -0.256 \\
\hline & \multirow[t]{3}{*}{ B } & $L C^{a}$ & 7.25 & $7.41-7.21$ & $7.31 \pm 0.0721$ & 0.987 & $100.854 \pm 0.995$ & 0.854 \\
\hline & & $I^{b}$ & 12.5 & $12.54-12.42$ & $12.48 \pm 0.0293$ & 0.236 & $99.884 \pm 0.235$ & -0.116 \\
\hline & & $H C^{c}$ & 17.75 & $17.98-17.75$ & $17.80 \pm 0.1267$ & 0.712 & $100.325 \pm 0.714$ & 0.325 \\
\hline & C & $L C^{a}$ & 5 & $5.04-4.95$ & $5.01 \pm 0.0188$ & 0.375 & $100.387 \pm 0.376$ & 0.387 \\
\hline & & $I^{b}$ & 10 & $10.15-9.99$ & $10.08 \pm 0.0269$ & 0.267 & $100.834 \pm 0.269$ & 0.834 \\
\hline & & $H C^{c}$ & 15 & $15.20-14.86$ & $15.03 \pm 0.0598$ & 0.398 & $100.256 \pm 0.399$ & 0.256 \\
\hline Ethanol & $A$ & $L C^{a}$ & 11.25 & $11.32-11.24$ & $11.29 \pm 0.0142$ & 0.126 & $100.423 \pm 0.126$ & 0.423 \\
\hline & & $I^{b}$ & 22.5 & $22.94-22.19$ & $22.69 \pm 0.1853$ & 0.817 & $100.856 \pm 0.823$ & 0.856 \\
\hline & & $H C^{c}$ & 33.75 & $33.71-33.48$ & $33.44 \pm 0.0913$ & 0.273 & $99.10 \pm 0.270$ & -0.897 \\
\hline & B & $\mathrm{LC}^{\mathrm{a}}$ & 16.25 & $16.41-16.15$ & $16.30 \pm 0.0950$ & 0.583 & $100.344 \pm 0.585$ & 0.344 \\
\hline & & $\mathrm{IC}^{\mathrm{b}}$ & 32.5 & $32.65-32.63$ & $32.64 \pm 0.0061$ & 0.019 & $100.435 \pm 0.019$ & 0.435 \\
\hline & & $H C^{c}$ & 48.75 & $49.48-48.55$ & $49.06 \pm 0.414$ & 0.846 & $100.643 \pm 0.851$ & 0.643 \\
\hline & C & $\mathrm{LC}^{\mathrm{a}}$ & 11.25 & $11.72-11.32$ & $11.34 \pm 0.0857$ & 0.756 & $100.823 \pm 0.762$ & 0.823 \\
\hline
\end{tabular}


Table 3 Accuracy and precision analysis data (Continued)

\begin{tabular}{|c|c|c|c|c|c|c|c|c|}
\hline \multirow[t]{2}{*}{ Solvent } & \multirow[t]{2}{*}{ Method } & \multirow[t]{2}{*}{ Level } & \multirow{2}{*}{$\begin{array}{l}\text { Conc } \\
-\mu \mathrm{g} / \\
\mathrm{ml}\end{array}$} & \multicolumn{3}{|c|}{ Estimated concentrations $\left(\mu \mathrm{g} \mathrm{ml}^{-1}\right)$ by linear regression equation } & \multirow{2}{*}{$\begin{array}{l}\text { Mean \% } \\
\text { recovery } \\
( \pm \text { S.D. })\end{array}$} & \multirow{2}{*}{$\begin{array}{l}\text { Accuracy } \\
d \\
(\% \text { RE) }\end{array}$} \\
\hline & & & & Range & A.M. \pm S.D. & $\%$ RSD & & \\
\hline & & $I C^{b}$ & 22.5 & $22.91-22.38$ & $22.67 \pm 0.1079$ & 0.476 & $100.765 \pm 0.479$ & 0.765 \\
\hline & & $\mathrm{HC}^{\mathrm{C}}$ & 33.75 & $33.93-33.58$ & $33.83 \pm 0.0669$ & 0.198 & $100.245 \pm 0.198$ & 0.245 \\
\hline \multirow[t]{9}{*}{ Methanol } & A & $\mathrm{LC}^{\mathrm{a}}$ & 7.5 & $7.63-7.34$ & $7.56 \pm 0.0308$ & 0.408 & $100.845 \pm 0.411$ & 0.845 \\
\hline & & $I C^{b}$ & 15 & $15.11-14.79$ & $14.95 \pm 0.0569$ & 0.381 & $99.67 \pm 0.379$ & -0.325 \\
\hline & & $H C^{c}$ & 22.5 & $22.93-22.10$ & $22.63 \pm 0.1527$ & 0.675 & $100.586 \pm 0.678$ & 0.586 \\
\hline & B & $\mathrm{LC}^{\mathrm{a}}$ & 10 & 10.19-9.89 & $10.08 \pm 0.0771$ & 0.765 & $100.847 \pm 0.771$ & 0.847 \\
\hline & & $I C^{b}$ & 20 & 20.40-19.64 & $20.01 \pm 0.1565$ & 0.782 & $100.097 \pm 0.782$ & 0.097 \\
\hline & & $H C^{c}$ & 30 & $30.53-29.59$ & $30.15 \pm 0.1957$ & 0.649 & $100.516 \pm 0.652$ & 0.516 \\
\hline & $C$ & $\mathrm{LC}^{\mathrm{a}}$ & 7.5 & $7.65-7.38$ & $7.55 \pm 0.0673$ & 0.891 & $100.784 \pm \mathrm{v} 0.897$ & 0.784 \\
\hline & & $\mathrm{IC}^{\mathrm{b}}$ & 15 & $15.22-14.76$ & $15.06 \pm 0.1211$ & 0.804 & $100.456 \pm 0.807$ & 0.456 \\
\hline & & $H C^{c}$ & 22.5 & $22.94-22.07$ & $22.47 \pm 0.2151$ & 0.957 & $99.903 \pm 0.956$ & -0.097 \\
\hline \multirow[t]{9}{*}{ DDW } & $A$ & $\mathrm{LC}^{\mathrm{a}}$ & 8.75 & $8.91-8.64$ & $8.80 \pm 0.0604$ & 0.686 & $100.658 \pm 0.690$ & 0.658 \\
\hline & & $I^{b}$ & 17.5 & $17.41-17.39$ & $17.42 \pm 0.0128$ & 0.074 & $99.548 \pm 0.073$ & -0.452 \\
\hline & & $H C^{C}$ & 22.5 & $22.64-22.39$ & $22.66 \pm 0.0423$ & 0.187 & $100.752 \pm 0.188$ & 0.752 \\
\hline & B & $\mathrm{LC}^{\mathrm{a}}$ & 10 & 10.14-9.91 & $10.00 \pm 0.097$ & 0.965 & $100.047 \pm 0.970$ & 0.047 \\
\hline & & $I C^{b}$ & 20 & $20.25-20.01$ & $20.10 \pm 0.083$ & 0.416 & $100.547 \pm 0.418$ & 0.547 \\
\hline & & $H C^{C}$ & 30 & $30.51-29.69$ & $30.07 \pm 0.2394$ & 0.797 & $100.243 \pm 0.798$ & 0.243 \\
\hline & C & $\mathrm{LC}^{\mathrm{a}}$ & 8.75 & $8.71-8.71$ & $8.71 \pm 0.0036$ & 0.043 & $99.65 \pm 0.042$ & -0.345 \\
\hline & & $I C^{b}$ & 17.5 & $17.83-17.36$ & $17.63 \pm 0.1510$ & 0.857 & $100.753 \pm 0.863$ & 0.753 \\
\hline & & $H C^{C}$ & 26.25 & $26.43-26.25$ & $26.36 \pm 0.0594$ & 0.475 & $100.435 \pm 0.477$ & 0.435 \\
\hline
\end{tabular}

${ }^{a}$ Lower concentration $(\mathrm{LC})=Q_{1}=$ value corresponding to rank $1 / 4(n+1)$ in $\mu \mathrm{g} \mathrm{ml}^{-1}$

${ }^{b}$ Intermediate concentration (IC) $=Q_{2}=$ value corresponding to rank $1 / 2(n+1)$ in $\mu \mathrm{g} \mathrm{ml}^{-1}$

'High concentration, corresponding to rank $3 / 4(n+1)$ in $\mu \mathrm{g} \mathrm{ml}^{-1}$

$\mathrm{d} \% R E=100 \times \frac{|\langle x\rangle-\mu|}{\mu} \cdot\left[\langle x\rangle=\right.$ avg of obs $\mathrm{HC}=\mathrm{Q}_{3}=$ value values, $\mu=$ central tendency of obs values $]$

${ }^{\mathrm{e}}$ Arithmetic mean $=\mathrm{AM}$

acetonitrile etc. Satranidazole (STZ) was found to dissolve at a good extent. The drug was very soluble in 1:4 DMF and dioxane-mixed solvents. The optical characteristics of satranidazole (STZ) in different solvent with their respective $\lambda_{\max }$ value were presented in Table 1 , and the absorbance profile of satranidazole was described in Fig. 1.

\section{Specificity and selectivity}

In the presence of common additives, UV-spectrum was not changed which reflected by the determination of $t$ values as it was found to be less than tabulated $t$ value in Table 2.

\section{Linearity}

Linearity ranges for satranidazole (STZ) estimation has been found to be very less for non-aqueous solvents, i.e., $5-10 \mu \mathrm{g} / \mathrm{ml}$ in case of acetonitrile unlike aqueous solvents, i.e., 5-30. Standard error value (S.E) of slope and intercept having a low amount which identified that the proposed method is highly precise and all values of mean slope and intercept within 95\% confidence interval limit. The high regression value was -0.999 in all the methods, and all the solvents (except methanol method A-0.998) shout for high quality of fit of regression coefficient values (Table 2).

\section{Accuracy}

The accuracy is shown within the linearity range in three different concentrations ( $\mathrm{HC}, \mathrm{IC}, \mathrm{LC})$ in all the solvents and all the methods. All the three proposed methods are found to have acceptable percentage recovery with RSD values below $1 \%$ which was described in Table 3. By the standard addition method, validity and reliability were farther assessed which was described in table 4.

\section{Precision}

All three methods were indicated by well precision results through repeatability $(\% \mathrm{RSD}<1)$ in a short interval of time. High intermediate precision reflects within day experiments both for drug products and drug substances $(\%$ RSD $<1)$ which was described in Tables 4 and 5 , respectively. 
Table 4 Summary of accuracy results by standard addition technique for pharmaceutical product form of satranidazole $($ STZ $)(n=10)$

\begin{tabular}{|c|c|c|c|c|c|c|}
\hline \multirow{3}{*}{$\begin{array}{l}\text { Solvent } \\
\text { used }\end{array}$} & \multirow{3}{*}{$\begin{array}{l}\text { Drug } \\
\text { taken } \\
(\mu \mathrm{g} \\
\left.\mathrm{ml}^{-1}\right)\end{array}$} & \multirow[t]{3}{*}{ Method } & \multicolumn{4}{|c|}{ \% Recovery ( \pm RSD) } \\
\hline & & & \multicolumn{4}{|c|}{ Pure drug added (recovery level) } \\
\hline & & & $0 \%$ & $80 \%$ & $100 \%$ & $120 \%$ \\
\hline \multirow[t]{3}{*}{ DD water } & 13 & A & $100.30 \pm 0.53$ & $100.91 \pm 0.04$ & $101.23 \pm 1.00$ & $99.97 \pm 0.49$ \\
\hline & & B & $99.85 \pm 0.61$ & $99.70 \pm 1.49$ & $98.95 \pm 1.36$ & $100.56 \pm 0.59$ \\
\hline & & C & $100.46 \pm 0.53$ & $100.43 \pm 1.24$ & $100.23 \pm 0.77$ & $100.49 \pm 0.56$ \\
\hline \multirow[t]{3}{*}{ Methanol } & 11 & A & $100.27 \pm 0.98$ & $100.15 \pm 0.15$ & $101.82 \pm 0.18$ & $100.16 \pm 0.74$ \\
\hline & & B & $101.36 \pm 0.18$ & $99.39 \pm 0.05$ & $101.86 \pm 0.81$ & $101.74 \pm 0.21$ \\
\hline & & C & $101.91 \pm 0.81$ & $99.84 \pm 0.05$ & $101.27 \pm 0.90$ & $100.41 \pm 0.83$ \\
\hline \multirow[t]{3}{*}{ Ethanol } & 18 & A & $99.61 \pm 0.45$ & $99.32 \pm 0.92$ & $100.00 \pm 0.72$ & $101.24 \pm 0.81$ \\
\hline & & B & $99.79 \pm 0.56$ & $101.23 \pm 1.24$ & $98.99 \pm 0.12$ & $100.43 \pm 0.68$ \\
\hline & & C & $100.44 \pm 0.16$ & $100.18 \pm 0.58$ & $99.86 \pm 0.04$ & $98.51 \pm 0.73$ \\
\hline \multirow[t]{3}{*}{ DMF } & 6 & A & $99.86 \pm 0.39$ & $100.28 \pm 0.37$ & $100.25 \pm 0.33$ & $99.09 \pm 0.45$ \\
\hline & & B & $99.58 \pm 0.50$ & $100.37 \pm 0.40$ & $98.92 \pm 0.42$ & $100.45 \pm 0.83$ \\
\hline & & $C$ & $100.25 \pm 0.17$ & $100.55 \pm 0.65$ & $98.90 \pm 0.83$ & $100.00 \pm 0.78$ \\
\hline \multirow[t]{3}{*}{ Methonol } & 11 & A & $99.97 \pm 0.32$ & $99.26 \pm 1.39$ & $100.94 \pm 1.60$ & $101.30 \pm 0.40$ \\
\hline & & B & $99.68 \pm 0.39$ & $101.90 \pm 1.00$ & $101.19 \pm 0.80$ & $100.83 \pm 1.27$ \\
\hline & & $C$ & $99.58 \pm 0.98$ & $100.00 \pm 0.00$ & $100.00 \pm 1.42$ & $100.63 \pm 0.89$ \\
\hline \multirow[t]{3}{*}{ Benzene } & 13 & A & $100.10 \pm 0.81$ & $99.97 \pm 0.03$ & $101.13 \pm 0.16$ & $99.56 \pm 0.07$ \\
\hline & & B & $100.42 \pm 0.82$ & $101.50 \pm 0.70$ & $99.58 \pm 1.10$ & $99.1 \pm 0.66$ \\
\hline & & $C$ & $99.89 \pm 0.06$ & $101.78 \pm 0.80$ & $100.50 \pm 0.20$ & $99.68 \pm 0.04$ \\
\hline \multirow[t]{3}{*}{ Acetonitrile } & 4 & A & $99.98 \pm 0.59$ & $100.13 \pm 0.24$ & $100.89 \pm 0.41$ & $101.13 \pm 0.01$ \\
\hline & & $B$ & $100.05 \pm 0.99$ & $100.44 \pm 0.16$ & $100.00 \pm 0.03$ & $100.4 \pm 0.09$ \\
\hline & & $C$ & $99.82 \pm 0.06$ & $100.06 \pm 1.60$ & $100.08 \pm 0.29$ & $100.9 \pm 0.02$ \\
\hline
\end{tabular}

*Percentage of relative standard deviation

Limit of detection (LOD) and limit of quantitation (LOQ) Highest LOD and LOQ found in methanol while STZ was estimated in method $C$ (3.7901 and 11.4084) and lowest (0.0002 and 0.0006) in DDW estimated using method A.

\section{Statistical evaluation}

Two-way ANOVA study was done (Table 6) to understand whether the differential effect of two factors, namely individual method and individual solvent exists (alternative hypothesis) or not (null hypothesis).

\section{Discussion}

In spectroscopy, organic solvents have shown either end-absorption below $220 \mathrm{~nm}$ due to $\mathrm{n} \rightarrow \Pi^{*}$ transition within their functional groups (ethanol, methanol, etc.) or specific absorption bands due to $\mathrm{n} \rightarrow \Pi^{*}$ transition (acetone, DMF, etc.) or $\Pi \rightarrow \Pi^{*}$ transition (benzene, toluene, etc.) within unsaturated groups. From the spectra of drug the sharp peak obtained within the region of 315$330 \mathrm{~nm}$ (most possibly due to $\mathrm{n} \rightarrow \Pi^{*}$ (R-band), transition of heteroaromatic group was chosen for diagnosis. The hyper-chromic shift of the R-band was observed in cases of DMF and acetonitrile possibly due to extensive secondary-bonding involving the lone pair of electrons on the nitrogen atom. The transition of polar bonds, like $\mathrm{C}=\mathrm{O}, \mathrm{S}=\mathrm{O}$, and $\mathrm{N}=\mathrm{O}$, is affected by solvent polarity. As solvent polarity increases, $\Pi \rightarrow \Pi^{*}$ bands undergo redshift, and $n \rightarrow \Pi^{*}$ bands undergo a blue shift [17]. The reason may be the more polar excited state is better stabilized relative to the ground state, in former case wherein latter case ground state with electron pair is more stabilized than an excited state with a single electron.

Here, in the absence of UV-spectrum, no statistically significant difference was found between mean absorbance of solutions in presence, and common excipients could be claimed which identified that the proposed analytical method is highly specific and selective for the drug [18].

The measurement of the range could be correlated with solubility. Hyper-chromic shift has been found in non-aqueous solvents, like DMF, acetonitrile, etc., and hypo-chromic shift has been found in aqueous solvent 
Table 5 Determination of satranidazole (STZ) from pharmaceutical product by spectrophotometric method

\begin{tabular}{|c|c|c|c|c|c|c|c|c|}
\hline Solvent & $\begin{array}{l}\text { Label } \\
\text { claimed } \\
\text { (mg) }\end{array}$ & Method & Amount of STZ found (mg/tab) & $\%$ Recovery \pm S.D. & $\%$ RSD & Accuracy ${ }^{a}(\%)$ & $\begin{array}{l}95 \% \text { C.L } \\
\text { of } t^{b}\end{array}$ & $\% \mathrm{RSD}$ \\
\hline \multirow[t]{3}{*}{ Double distilled water } & \multirow[t]{3}{*}{300} & A & $298.488 \pm 0.117$ & $99.496 \pm 0.039$ & 0.039 & -0.504 & 2.19 & 0.023 \\
\hline & & $\mathrm{B}$ & $299.349 \pm 0.123$ & $99.783 \pm 0.041$ & 0.041 & -0.217 & 1.53 & 0.024 \\
\hline & & C & $298.92 \pm 0.294$ & $99.64 \pm 0.098$ & 0.098 & -0.36 & 1.95 & 0.581 \\
\hline \multirow[t]{3}{*}{ Methanol } & \multirow[t]{3}{*}{300} & A & $302.454 \pm 0.141$ & $100.81 \pm 0.047$ & 0.046 & 0.818 & 1.36 & 0.028 \\
\hline & & B & $301.848 \pm 2.91$ & $100.61 \pm 0.970$ & 0.964 & 0.616 & 1.91 & 0.577 \\
\hline & & C & $302.115 \pm 2.601$ & $100.70 \pm 0.867$ & 0.860 & 0.705 & 2.11 & 0.516 \\
\hline \multirow[t]{3}{*}{ Ethanol } & \multirow[t]{3}{*}{300} & A & $302.058 \pm 2.481$ & $100.68 \pm 0.806$ & 0.821 & 0.686 & 2.14 & 0.492 \\
\hline & & B & $301.917 \pm 2.16$ & $100.63 \pm 0.720$ & 0.715 & 0.639 & 2.16 & 0.428 \\
\hline & & C & $300.804 \pm 2.385$ & $100.26 \pm 0.795$ & 0.792 & 0.268 & 1.01 & 0.473 \\
\hline \multirow[t]{3}{*}{ DMF } & \multirow[t]{3}{*}{300} & A & $301.701 \pm 2.889$ & $100.56 \pm 0.963$ & 0.957 & 0.567 & 1.77 & 0.573 \\
\hline & & B & $301.803 \pm 1.911$ & $100.60 \pm 0.637$ & 0.633 & 0.601 & 2.06 & 0.379 \\
\hline & & C & $301.887 \pm 0.225$ & $100.62 \pm 0.075$ & 0.074 & 0.629 & 2.15 & 0.045 \\
\hline \multirow[t]{3}{*}{ Benzene } & \multirow[t]{3}{*}{300} & A & $301.971 \pm 1.083$ & $100.65 \pm 0.361$ & 0.358 & 0.657 & 1.39 & 0.215 \\
\hline & & B & $300.861 \pm 2.754$ & $100.28 \pm 0.918$ & 0.915 & 0.287 & 0.94 & 0.546 \\
\hline & & C & $300.204 \pm 0.084$ & $100.06 \pm 0.028$ & 0.027 & 0.068 & 1.77 & 0.017 \\
\hline \multirow[t]{3}{*}{ Acetonitrile } & \multirow[t]{3}{*}{300} & A & $297.540 \pm 1.419$ & $99.180 \pm 0.473$ & 0.476 & -0.820 & 1.26 & 0.281 \\
\hline & & $B$ & $297.507 \pm 2.124$ & 99. $169 \pm 0.708$ & 0.713 & -0.831 & 1.33 & 0.421 \\
\hline & & C & $297.174 \pm 2.028$ & $99.058 \pm 0.676$ & 0.682 & -0.942 & 1.82 & 0.402 \\
\hline \multirow[t]{3}{*}{ Dichloro methane } & \multirow[t]{3}{*}{300} & A & $299.034 \pm 2.043$ & $99.678 \pm 0.681$ & 0.683 & -0.322 & 1.41 & 0.405 \\
\hline & & B & $297.504 \pm 2.844$ & 99. $168 \pm 0.948$ & 0.955 & -0.832 & 2.20 & 0.563 \\
\hline & & C & $299.769 \pm 1.08$ & $99.923 \pm 0.360$ & 0.360 & -0.077 & 0.64 & 0.214 \\
\hline
\end{tabular}

$\mathrm{a}_{\%} \%$ relative error $=100 * \frac{\text { Conc }_{\text {predicted }}-\text { Conc }_{\text {normal }}}{\text { Conc normal }}$

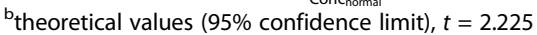

'Intra-day RSD value

[19]. It could be explained by the degree of secondary bonding which is proportional to solubility and reciprocal to linearity due to high absorptivity.

All the solvents were utilized yelling for high accuracy of scientific technique. The legitimacy and unwavering quality of proposed strategies are evaluated by recovery studies. Accuracy was found to be between $\pm 1 \%$ in every case. The \% RSD is found to be less than $2 \%$ in all the cases $(80 \%, 100 \%$, and $120 \%)$, and accuracy was found to be between $\pm 2 \%$ in all the cases [20]. These outcomes uncovered that any little change in the medication focus in the arrangement could be precisely dictated by the proposed analytical methods.

Table 6 Results of two ways analysis of variance (ANOVA)

\begin{tabular}{lll}
\hline SL no. & Comparison & $F$ value (tabulated) \\
\hline 1. & Method A vs method B & $F_{0.05}^{\text {Tabu }}(2,12)=3.89$ \\
2. & Method A vs method C & $F_{0.05}{ }^{\text {Tabu }}(2,6)=3.85$ \\
3. & Method C vs method B & $F_{0.05}{ }^{\text {Tabu }}(6,12)=3.87$ \\
\hline
\end{tabular}

Precision was dictated by considering the repeatability and transitional accuracy. All techniques have phenomenal repeatability and intermediate precision in all the solvent utilized. They were determined under Student's $t$ value which does not exceed the tabulated $t$ value [21]. Limit detection and limit quantification measurements were seen as totally different in various solvents.

In individual methods effect determined are equal and with no significant differences at $95 \%$ confidence level as observed F0.05 $(2,12)<$ tabulated $F 0.05(2,12)$ which was further supported by the difference between any two methods observations are always lower than the critical difference value calculated. But individual solvents effect determined are not equal and with significant differences at $95 \%$ confidence level as observed $F 0.05(6,12)>$ tabulated $F 0.05(6,12)$ [21]. Critical difference value is used to compare with all pairs of samples to sample total and the difference between DDW and acetonitrile was found to be greater than the critical difference value. So, there was no significant effect of different solvents except 


\section{STANDARDE CURVE OF STZ IN DIFFERENT SOLVENTS}

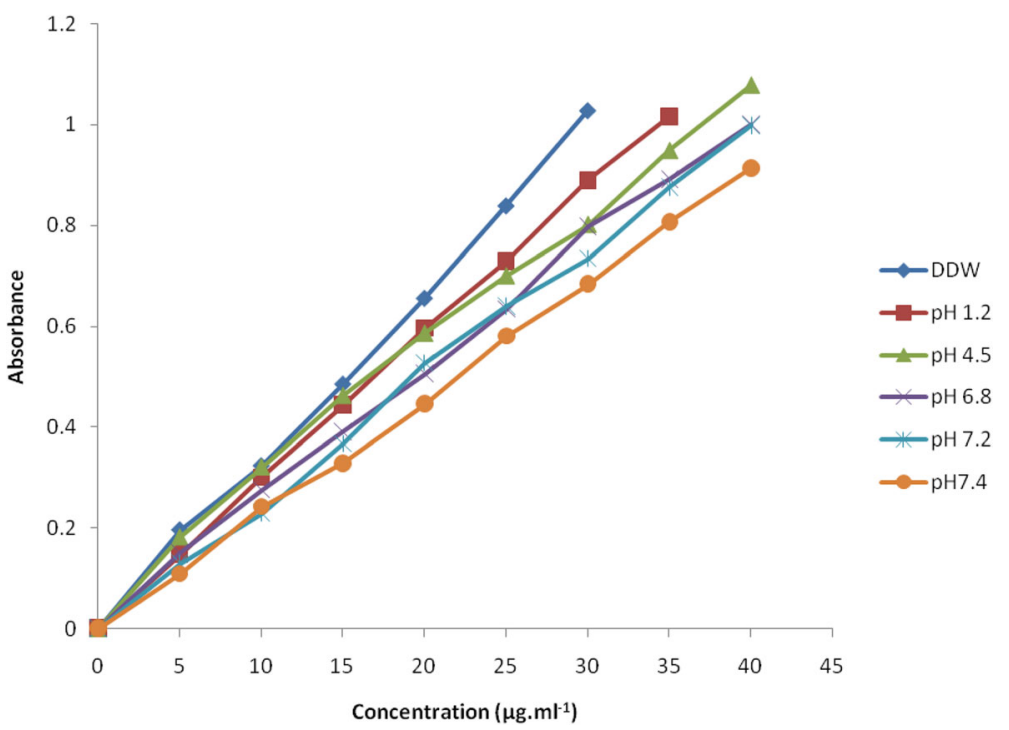

Fig. 1 Graphical representation of linear curves of satranidazole

DDW and acetonitrile.Graphical representation of linear curves of satranidazole

\section{Conclusion}

Three different UV-spectrophotometric methods were developed for satranidazole (STZ) determination in various solvents ranging from inorganic to organic solvents. The analytical method was found to be simple, sensitive, rapid, clear, and economic, and it may be utilized for the normal examination, quality control, medicate entanglement effectiveness, and discharge concentrate from any pharmaceutical measurements process. All the three methods could be used equivalently to analyze STZ samples.

\section{Abbreviations}

STZ: Satranidazole; DDW: Double distilled water; ICH: International Conference for Harmonization; AUC: Area under curve; LOD: Limit of detection; LOQ: Limit of quantification

\section{Acknowledgements}

The authors wish to give thanks to the School of Pharmacy, Techno India University, EM 4, Sector-V, Kolkata-700091, West Bengal, India, and Calcutta Institute of Pharmaceutical Technology for their constant support and for giving research laboratory to carry out this project work. We would also like to thank our fellow colleagues for giving help and encouragement in completing the research project work.

\section{Authors' contributions}

We declare that this work was done by the authors named in this article. $\mathrm{RM}, \mathrm{BM}$, and RNP conceived and designed the study. RM and ADC carried out the laboratory work, collected and analyzed the data. SM drafted the manuscript. BM supervised the work, and RM assisted in the data analysis. All authors have read and approved the final manuscript.

\section{Funding}

The authors have no funding to report.
Availability of data and materials

All necessary data generated or analyzed during this study are included in this published article. Any additional data could be available from the corresponding author upon request.

Ethics approval and consent to participate Not applicable.

\section{Consent for publication}

Not applicable.

\section{Competing interests}

The authors declare that they have no competing interests.

Received: 12 May 2020 Accepted: 6 August 2020

Published online: 18 August 2020

\section{References}

1. Pawar HA, Joshi PR (2014) Development and validation of a discriminating in-vitro dissolution method for oral formulations containing satranidazole. Inter J Spectro 1:1-7

2. Barry CE, Boshoff HIM, Dowd CS (2004) Prospects for clinical introduction of nitroimidazole antibiotics for the treatment of tuberculosis. Current Pharma Desig 10:3239-3262

3. Muzaffar J, Madan K, Sharma MP, Kar P (2006) Randomized, single-blind, placebo-controlled multicenter trial. Dig Dis Sci 51(12):2270-2273

4. Shinde RS, Bhoir IS, Pawar SN, Yadav BS, Bhagwat MA (2010) Simultaneous estimation of satranidazole and ofloxacin in tablet dosage form by high performance liquid chromatography. E J Chem 7(1):198-202

5. Patel MB, Patel KM, Patel GS, Suhagia BN, Prajapati AMV (2007) Development and validation of a stability-indicating HPTLC-densitometric method for Satranidazole. J Liq Chromatogr Rel Technol 30:2459-2471

6. Lalla J, Hamrapurkar P, Anu R, Wadhwa T (2003) Highperformance thin-layer chromatographic determination of Satranidazole in its dosage form. JPC- J Planar Chromatogr Modern TLC 16:447-450

7. Reddy KPR, Prathap KMS, Sharma H, Kumar KV (2019) A simple colorimetric method for the determination of raloxifene hydrochloride in pharmaceuticals using modified romini's reagent. Inter J Anal Chem 1:1-5

8. Aderibigbe SA, Adegoke OA, Idowu OS (2012) A new colorimetric method for the determination of nifedipine tablets by derivatization using 4carboxyl-2,6-dinitrobenzene diazonium ion. Inter J Indus Chem 3(5):1-8 
9. Kolachina V, Saha RN (2005) New, simple and validated UVspectrophotometric methods for the estimation of gatifloxacin in bulk and formulations. Farmaco 60(11-12):906-912

10. Game MD, Sakarkar DM (2011) Simultaneous spectrophotometric estimation of nitazoxanide and ofloxacin in tablets. Ind J Pharm Sci 73(1):70-74

11. Route AR, Bhalerao SR (2011) First order derivative spectrophotometric estimation of nabumetone and paracetamol in tablet dosage form. Pharm Methods 2(4):260-263

12. Bhoir SJ, Gaikwad PV, Parab LS, Shringarpure RN, Savant SS, Verma PJ (2011) RP-HPLC method development and validation for the simultaneous estimation of satranidazole and ofloxacin in pharmaceutical dosage form. J Chrom Sci 49:1-4

13. Bansal K, Rawat MK, Jain A, Rajput A, Chaturvedi TP, Singh S (2009) Development of satranidazole mucoadhesive gel for the treatment of periodontitis. AAPS Pharm Sci Tech 10(3):716-723

14. Castrol GT, Blanco SE, Giordano OS (2000) UV spectral properties of benzophenone influence of solvents and substituents. Molecules 5:424-425

15. Sahoo PK, Sharma R, Chaturvedi SC (2008) Simultaneous estimation of metformin hydrochloride and pioglitazone hydrochloride by RPHPLC method from combined tablet dosage form. Ind J Pharm Sci 70(3):383-386

16. International conference for harmonization (ICH) (1995) Validation of analytical procedures: text and methodology. Euro Med Agency Q2(R1):1-15

17. Adeogun Al, Odozi NW, Obiegbedi NO, Bello OS (2008) Solvents effect on $n \rightarrow \pi^{*}$ and $\pi \rightarrow \pi^{*}$ transition of 9- fluorenone. Afric J Biotech 7(15):2736-2738

18. Desai VN, Afieroho OE, Dagunduro BO, Okonkwo TJ, Ndu CC (2011) A simple uv spectrophotometric method for the determination of levofloxacin in dosage formulations. Trop J pharma Resear10 (1): 75-79.

19. Uliana CV, Garbellini GS, Yamanaka H (2012) Spectrophotometric evaluation of the behavior of disperse red 1 dye in aqueous media and its interaction with calf thymus ds-DNA. J Braz Chem Soc 23(8):1469-1475

20. Chhajed SS, Sonawane S, Pingle AP, Dashputre N, Sakshi KSJ (2019) Development and validation of uv spectrophotometric method for estimation of pregabalin. Asian J Pharm Ana 9(1):15-18

21. Fraihat SM, Bahgat KM (2014) Spectrophotometric methods for the determination of ketoconazole in pharmaceutical dosage forms. Trop J Pharma Resear 13(9):1511-1514

\section{Publisher's Note}

Springer Nature remains neutral with regard to jurisdictional claims in published maps and institutional affiliations.

\section{Submit your manuscript to a SpringerOpen ${ }^{\circ}$ journal and benefit from:}

- Convenient online submission

- Rigorous peer review

- Open access: articles freely available online

- High visibility within the field

- Retaining the copyright to your article

Submit your next manuscript at $\boldsymbol{\nabla}$ springeropen.com 